\title{
Overview on Head Posture Valuation from a 2D Face Image
}

\author{
Seema N. Shaikh ${ }^{1}$, Sushil Kumar N. Holambe ${ }^{2}$ \\ Persuing ME (CSE), College of Engineering, Osmanabad, India \\ PG Coordinator ME (CSE), College of Engineering, Osmanabad, India
}

\begin{abstract}
The common human capability is to estimate the pinnacle cause of another person. This capability of human presents a unique challenge for laptop vision systems. During this paper it's pictured the thanks to estimate head cause angles from second face image using a 3D face model morphed from a reference face model. A reference model points to $3 D$ face of a private of constant background and build the question subject. The projected theme minimizes the excellence between the two sets of projected face expression on the question face image and additionally the corresponding points on the $3 D$ face model to estimate the pinnacle cause angles. The 3D face model used is morphed from a reference model to be extra specific to the question face in terms of the depth error at the feature points. A 3D face model is made by the morphing technique which may be extra specific to the question image once multiple second face footage of the question discipline unit accessible for coaching job. The projected morphing technique is computationally effective as a result of the depth of a 3D face model is adjusted by a scalar depth parameter feature points. Best depth parameters area unit units found by minimizing the excellence between the second choices of the question face image and additionally the corresponding choices on the morphed $3 D$ model projected onto the second face.
\end{abstract}

Keywords: 3Dfacemodel, depth valuation, feature in equality minimization, Head postur evaluation, altering.

\section{Introduction}

Head cause angles area unit the units calculated with the high sensitivity capability of human operator to a specific object among the environment. From an early age, people can merely and quickly understand the orientation and movement of caput, which allow one to know the intentions of others World Health Organization area unit shut among the sort of non-verbal communication. but this task creates the matter that has challenged machine systems for several years. during a laptop vision perspective, head cause estimation is the tactic of considering the orientation of somebody's head from a information storage. Estimating head cause angles from second face model is hard as a result of the scant knowledge provided to the system among the information storage. It desires a series of method steps to transform a pixel-based illustration of a head into a notion of direction. Like completely different facial vision method steps, an ideal head cause laptop ought to demonstrate changelessness to a variety of image-changing factors. These factors embody physical phenomena like camera alteration, geometry, multisource softly glowing lighting, additionally as biological look, countenance, and additionally the presence of accessories like glasses and hats.

In the perspective of laptop vision, head cause estimation is most commonly understood as the ability to complete the orientation of a person's head relative to the vision of a camera. Extra strictly, head cause estimation is that the ability to complete the orientation of a head relative to a world arrangement, but this delicate distinction desires data of the intrinsic camera parameters to undo the activity bias from perspective distortion.

In laptop vision head cause estimation is very energetic investigation since it's a lot of importance in many applications such as face recognition, eye gaze following, and human-computer interaction. In face recognition, dissimilarities in head cause additionally in illumination changes can significantly decrease the performance of face recognition. Gaze estimation could be a important application in head cause estimation. throughout this the gaze direction is set by a mix of the eccentricity of the pupil center from the eye center and additionally the pinnacle cause angles. Head cause estimation could also be done from a second face image by two ways. These approaches area unit a unit appearance-based and feature-based. In appearance-based approaches, the strive is made to match somewhat of the image containing the face to similar face footage among the information to estimate the pinnacle cause. throughout this approach, head cause estimation becomes a pattern classification issue. look primarily based techniques work with low-resolution footage, but a finite vary of predefined cause angles could also be countable. One of major challenges with this head cause estimation theme is that this technique wishes to gather a adequate amount of coaching job information uniformly distributed across varied cause angles. Their performance might decrease considerably once the input face image contains variations like background, lighting, countenance, age, and identity. Feature-based approaches estimate the pinnacle cause from communications between the choices of a face image and of on a $3 \mathrm{D}$ face model. Feature-based approaches understand realistically actual head cause estimates for all three head cause angles, i.e., nodding, shaking, and tilting. the pinnacle cause is countable by the quantity of rotation throughout the fitting technique. The performance depends on the accuracy of a $3 \mathrm{D}$ face model representing the subject. Feature-based ways usually would like a computationally costly fitting technique as extra 3D model templates unit added to the set of example 3D models. A Generic Elastic Model (GEM) reconstructs a $3 \mathrm{D}$ face model from a second face image using a generic 3D model of constant gender and quality as a result of the question subject. 


\section{International Journal of Science and Research (IJSR) \\ ISSN (Online): 2319-7064}

Index Copernicus Value (2013): 6.14 | Impact Factor (2015): 6.391

In this paper, the projected methodology uses a little low vary of outstanding face expression like eye corners, nose tip, and lip corners extracted from the question face image. AN automatic feature extraction methodology is used to find the key points. Active look Model (AAM) has been applied to use the tactic. During this methodology, the feature points were selected manually on a second face image and mapped onto a reference 3D face model for analysis purpose. Head cause angles unit projected by minimizing the difference between the choices on the question face image and additionally the corresponding points on the $3 \mathrm{D}$ face model projected onto the second house. If there unit some cases where there unit extra face footage with acknowledged cause of the question discipline unit accessible, the reference 3D model could also be morphed for extra correct model fitting. The advanced 3D face model becomes extra specific to the question subject in terms of depth errors at the feature points. This morphing technique involves multiplying a scalar depth parameter to the depth of a reference 3D face model at each feature purpose. Best depth parameters area unit found by minimizing the excellence between \{the choices the choices of the second question face image and additionally the corresponding options on the morphed $3 \mathrm{D}$ model projected onto the second house. The projected morphing technique uses the simplest depth parameters therefore it's computationally economical the use of depth parameters simplifies the morphing technique to allow native twist at each facial feature purpose for proper $3 \mathrm{D}$ model building. AN best set of depth parameters unit found by minimizing the difference between \{the choices the choices\} of the question second face image and additionally the corresponding options of the morphed $3 \mathrm{D}$ model projected onto the second face. Throughout this paper projected head cause estimation technique was evaluated on two benchmarking information's: 1) the USF Human-ID info for depth estimation and 2) the inform information for head cause estimation.

Most of the times, people use the orientation of their heads to specific loaded, social, non-verbal information. As an example, somebody will purpose the direction of his head to indicate World Health Organization is that the planned object of a language. There could be a crucial which means among the movement of the highest as a method of check in AN extremely language. people nod to purpose that they understand what is being same and they use additional signals to purpose opposition, confusion, consideration and agreement. Inflated head movements unit similar with inform a finger, that is ready to point someone to look at a specific location. If two people center their visual attention on each other, usually spoken as mutual gaze, usually this will be usually a sign that two people unit engaged in an extremely discussion. Mutual gaze may additionally be used as a sign of awareness, e.g., a prosaic will wait for a stopped racer to look at him before getting in a crossover. A person's head direction may additionally provide information relating to the setting. If somebody shifts his head toward a selected direction, it indicates Associate in Nursing object of interest. Like speech recognition, head produce estimation will in all probability become Associate in Nursing customary tool to tie the gap between humans and laptop computer

\section{Planned System}

The planned morphing technique is computationally economical since the depth of the 3D reference model is meaning by the depth at each feature purpose increased by a depth parameter. The use of depth parameters simplifies the morphing technique to allow native twist at each facial feature purpose for proper 3D model building. The foremost effective set of depth parameters unit of measurement found by minimizing the excellence between choices the choices of the question second face image and conjointly the corresponding options of the morphed $3 \mathrm{D}$ model projected onto the second space. A projection matrix is used to relate the coordinates of a degree among the $3 \mathrm{D}$ space to its projection onto the second image plane. This technique is used among the rotation scale translation camera model. Usually in many wise things a 3D face model of the subject is not offered, then in such cases a 3D face model of a theme matter of a similar sex and origin as a result of the question subject is chosen as a reference model. Simply just in case lots of second face footage with known produce of the question subject unit of measurement offered, the depth of the reference face model is also refined through a 3D morphing technique to spice up the accuracy of produce estimation. The reference 3D face model is turned by the produce angles of the given employment image to reduce the difference between the second and conjointly the projected 3D feature vectors. The abstraction deviations $\mathrm{dX}$ and metal unit of measurement found once regulation of the second and 3D feature points. These abstraction deviations units of measurement significantly very little compared to the depth variation $\mathrm{dZ}$.

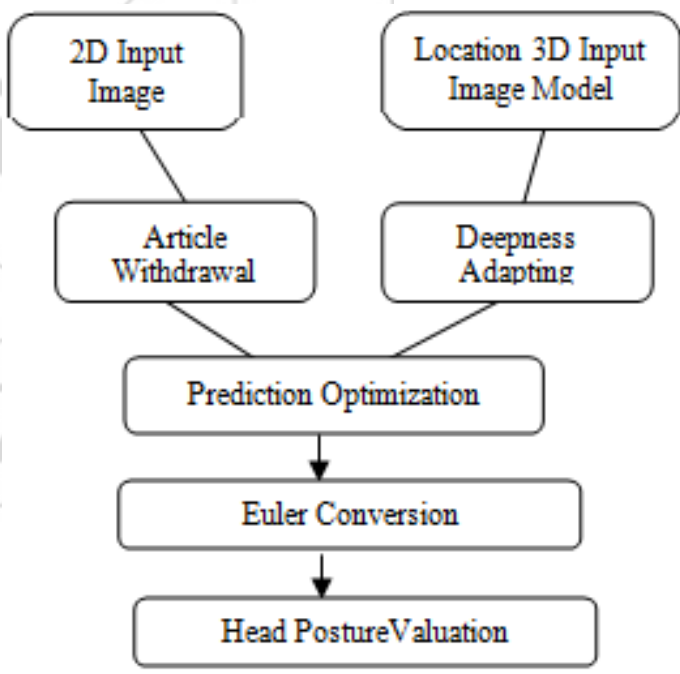

Figure 1: Basic Diagram of Planned System

\section{Head Posture valuation Ways}

There are varied ways that for head posture valuation. It's a challenge to arrange all the ways that into one catalog. The catalog consists of following categories that describe the approaches want to evaluate the top cause:

\section{A. Form example ways that :}

These ways that use image-based comparison metrics to match a vision of a person's head to a gaggle of examples with corresponding produce tags. The templates are also 


\section{International Journal of Science and Research (IJSR) \\ ISSN (Online): 2319-7064 \\ Index Copernicus Value (2013): 6.14 | Impact Factor (2015): 6.391}

swollen to an even bigger set at any time that is ready to change systems to adapt to propellent conditions. Negative employment examples do not appear to be required throughout this instance ways that. Associate in Nursing amount of employment data desires only cropping head footage and providing head create observations. Look templates unit of measurement to boot well fitted to every high and low-resolution basic cognitive process.

There are some disadvantages with this method. Whereas not the use of some interruption technique, they are only capable of valuing separate produce locations. They often assume that the head region has already been detected and localized, and localization error can degrade the accuracy of the highest produce estimate. They'll to boot suffer from efficiency issues, since as lots of templates unit of measurement else to the instance set, lots of computationally expensive image comparisons will wish to be computed. One answer to these last a pair of problems was to train a set of Support Vector Machines (SVMs) to search out and localize the face, and later use the support vectors as look templates to estimate head produce.

\section{B. Sensor Ranges}

Sensor ranges operate directly on an image house. The image is estimated by a sensor trained on many footage by using a supervised learning rule.

An advantage of sensor ranges ways that is that a separate head detection and localization step is not required, as a results of each detector is capable of constructing the excellence between head and nonhead. Synchronized detection and build estimation can be performed by applying the detector to many sub sections among the image. Detector arrays work employment algorithms that learn to overlook the appearance variation that does not correspond to form change. Sensor ranges unit of measurement to boot well fitted to high and low-resolution footage.

Disadvantages of this method are that it's robust to educate many detectors for each separate produce. For a detector array to perform as a head detector and build skilled, it ought to even be trained on many negative nonface examples that require significantly lots of employment data. There would possibly arise systematic problems as a result of the range of detectors is accumulated. If a pair of detectors unit of measurement united to really similar poses, the photographs that unit of measurement positive employment examples for one ought to be negative employment examples for a new.

\section{Nonlinear Reversion Policies:}

Nonlinear reversion policies assess produce by learning a nonlinear helpful mapping from the image house to a minimum of one or further produce directions. The limitation with these approaches is that it isn't clear but well a specific regression tool ar able to learn the correct mapping.

The high spatiality of Associate in Nursing image presents a challenge for a couple of regression tools. Support Vector Regressors (SVRs) are going to be used successfully if the spatiality of the data can be reduced.
An example of this method is multilayer perceptron (MLP) that consists of the various feed-forward cells made public in multiple layers. The perceptron is trained by backpropagation that might be a supervised learning procedure that propagates the error backward through each of the hidden layers among the network to update the weights and biases. Head produce can be expected from cropped footage of a head victimization Associate in Nursing MLP in various configurations.

There are varied advantages of neural network approaches. These systems ar terribly fast. They only would like cropped labelled faces for employment, work well in near-field and far-field basic cognitive process, and supply some of the most correct head produce estimates in follow.

The disadvantage to those methods is that they are disposed to error from poor head localization. A solution to the present is, a convolutional network that extends the MLP by expressly modeling some shift, scale, and distortion changelessness are going to be wont to reduce this offer of error.

\section{Multipurpose Models}

In multipurpose models, a nonrigid model is appropriate the image such that it follows to the facial structure of each individual. to boot to form labels, these methods would like employment data with explained facial features, but it permits them to create evaluations at the feature level rather than at the planet look level.

Active look Model (AAM) is that the model developed for head produce estimation that learns the primary modes of variation in facial type and texture from a second viewpoint. To form Associate in Nursing AAM, first a filled with organic structure Model (ASM) ought to be generated from a set of employment data. Then the face footage ought to be changed such that the feature points match those of the mean type. The changed footage need to be normalized and then used to build a shape-free texture model. At last, the correlations between type and texture ar learned and used to generate a combined look model. If a rough format of face form is given, the AAM can be fitted to a new facial image by iteratively comparison the extracted look model to the observed image and adjusting the model parameters to attenuate a distance live between these a pair of footage. Once the model has met to the feature locations, Associate in Nursing estimate of head produce are going to be obtained by mapping the appearance parameters to a produce estimate.

\section{Experiment Results}

To evaluate the performance of the planned head produce estimation technique, we've an inclination to use the mean absolute error (MAE) of drooping and shaking angles of head produce as a result of the analysis metric. The produce estimation error is computed by averaging the excellence between the ground-truth and conjointly the estimated produce angles for all footage. 


\section{International Journal of Science and Research (IJSR) \\ ISSN (Online): 2319-7064}

Index Copernicus Value (2013): 6.14 | Impact Factor (2015): 6.391

\section{Conclusion}

This paper presents head produce estimation from a single second face image using a 3D face model morphed from a reference 3D face model of somebody of identical origin and sex as a result of the question subject. Head produce angles ar estimated by minimizing the excellence between the choices on the question face image and conjointly the corresponding points on the $3 \mathrm{D}$ face model. once multiple footage of the question subject ar on the marketplace for employment, the reference model are going to be morphed for added correct model fitting. The advanced 3D face model becomes further specific to the question face image in terms of depth errors at the feature points. The planned morphing technique is computationally economical since the 3D depth is adjusted by multiplying the depth at each feature purpose by a scalar depth parameter. Experiments with the USF Human-ID data counsel that morphed 3D face model decreases depth errors equally as feature difference. Sex and origin variations between check subject and 3D model have an impression on the performance of head produce estimation.

\section{References}

[1] H.-S. Kooand K.- M. Lam, " Recovering the3D shape and poses of face images based on the similarity transform, " Pattern Recognit. Lett., vol. 29, no.6, pp.712-723, Apr.2008.

[2] R. Valenti, N. Sebe, and T. Gevers, "Combining head pose and eye location in formation for gaze estimation, "IEEE Trans. Image Process., vol.21, no.2, pp.802-815, Feb.2012.

[3] R. O. Mbouna, S. G. Kong, and M.- G. Chun, "Visual analysis of eye state and head pose for driver alertness monitoring, " IEEE Trans. Intell. Transp.Syst., vol.14, no.3, pp.1462-1468, Sep.2013.

[4] E. Murphy-Chutorian and M. M. Trivedi, "Head pose estimation in computer vision: A survey, "IEEE Trans.PatternAnal.Mach.Intell., vol.31, no.4, pp.607626, Apr.2009.

[5] S. Yan, H. Wang, J. Tu, X. Tang, and T. S. Huang, "Mode-kn factor analysis for imageensembles, "IEEETrans.ImageProcess., vol.18, no.3, pp.670-676, Mar.2009.

[6] V. N. Balasubramanian, J. Ye, and S. Panchanathan, "Biasedmanifold embedding: A framework for personindependent head pose estimation," in Proc. IEEE Conf. Comput. Vis. Pattern Recognit., Jun.2007, pp.1-7.

[7] R.Stiefelhagen, "Estimating head pose with neural networks-Results on the Pointing 04 ICPR workshop evaluationdata, "inProc.IEEEIntConf.PatternRecognit., Aug.2004, pp.8-11.

[8] D.Huang, M.Storer, F. Dela Torre, and H.Bischof, "Supervised local subspace learning for continuous head pose estimation, "in Proc. IEEE Conf.Comput.Vis.PatternRecognit., Jun.2011, pp.29212928.

[9] C. Tomasi and T. Kanade, "Shape and motion from image streams under orthography: A factorization method," Int. J. Comput. Vis., vol.9, no.2, pp.137-154, 1992.
[10] C. Bregler, A. Hertzmann, and H.Biermann, "Recovering non-rigid 3D shape from image streams," in Proc. IEEE Conf. Comput. Vis. Pattern Recognit., vol.2.Jun.2000, pp.690-696.

[11]D.Jelinek and C. J. Taylor, "Reconstruction of linearly parameterized models from single images with a camera of unknown focal length, " IEEE Trans.Pattern Anal.Mach.Intell., vol.23, no.7, pp.767-773, Jul.2001.

[12]Z.-L.Sun, K.-M.Lam, and Q.- W. Gao, "Depthestimation of face images using the non linear least-squares model, "IEEE Trans. Image Process., vol.22, no.1, pp.17-30, Jan.2013.

[13] P. Martins and J. Batista, "Single view head pose estimation," inProc. $15^{\text {th }}$ IEEE Int. Conf. Image Process., Oct.2008, pp.1652-1655.

[14] J.Xiao, S.Baker, I.Matthews, andT.Kanade, "Realtimecombined 2D+3D active appearance models, "in Proc. IEEE Conf. Comput.Vis.Pattern Recognit., vol.2.Jun. 2004, pp.535-542.

[15] J.Gonzalez-Mora, F.DelaTorre, N.Guil, and E.L.Zapata, "Learning a generic 3D face model from 2D image databases using incre- mental structure-from-motion," Image Vis. Comput., vol. 28, no. 7, pp.1117-1129, Jul.2010.

[16] V. BlanzandT. Vetter, "Face recognition based on fitting a 3D morphable model,'IEEETrans. Pattern Anal. Mach. Intell., vol.25, no.9, pp.1063-1074, Sep.2003.

[17] N.Gourier, J.Maisonnasse, D.Hall, and J.L. Crowley, "Headpose estimation on low resolution images," in Multimodal Technologies for Perception of Humans (Lecture Notes in Computer Science), vol.4122. Berlin, Germany:Springer-Verlag, 2007, pp.270-280.

[18] J. M. Blain, TheComplete Guide to Blender Graphics: Computer ModelingandAnimation.BocaRaton, FL, USA:CRCPress, 2012.

[19] J. Tu, Y. Fu, andT. S. Huang, "Locating nose-tips and estimating head posesin images by tensor poses, "IEEE Trans. Circuits Syst. Video Technol., vol.19, no.1, pp.90102, Jan. 2009.

[20] M. Fenzi, L. Leal-Taixe, B. Rosenhahn, andJ. Ostermann, "Class generative models based on feature regression for pose estimation of object categories, " in Proc. IEEE Conf. Comput.Vis.Pattern Recognit., Jun.2013, pp.755-762.

[21]I.Matthews and S.Baker, "Active appearance models revisited, "Int. J.Comput.Vis., vol.60, no.2, pp.135-164, 2004.

[22] J.Heo and M. Savvides, "Gender and ethnicity specific generic elastic models from a single 2D image for novel $2 \mathrm{D}$ pose face synthesis and recognition, "IEEETrans.PatternAnal.Mach.Intell., vol.34, no.12, pp.2341-2350, Dec.2012.

[23] G.GuoandG.Mu, "A study of large-scale ethnicity estimation with gender and age variations," in Proc. IEEE Comput.Soc.Conf.Comput. Vis.PatternRecognit., Jun.2010, pp.79-86.

[24]H. Hanand A. K.Jain, "Age, gender and race estimation from uncon- strained face images,'Dept. Comput.Sci.Eng., MichiganStateUniv., EastLansing, MI, USA, MSUTech.Rep.(MSU-CSE-14-5), 2014. 